\title{
A simple correction for the parallax effect in X-ray pair distribution function measurements
}

\section{Frederick Marlton, Oleh Ivashko, Martin v. Zimmerman, Olof Gutowski, Ann-Christin Dippel and Mads Ry Vogel Jørgensen}

J. Appl. Cryst. (2019). 52, 1072-1076

\section{IUCr Journals CRYSTALLOGRAPHY JOURNALS ONLINE}

Copyright (C) International Union of Crystallography

Author(s) of this article may load this reprint on their own web site or institutional repository provided that this cover page is retained. Republication of this article or its storage in electronic databases other than as specified above is not permitted without prior permission in writing from the IUCr.

For further information see http://journals.iucr.org/services/authorrights.html 


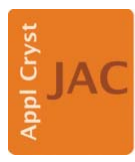

JOURNAL OF

APPLIED

CRYSTALLOGRAPHY

ISSN 1600-5767

Received 10 May 2019

Accepted 20 August 2019

Edited by K. Chapman, Stony Brook University, USA

Keywords: total scattering; pair distribution function; X-rays; parallax effect.

Supporting information: this article has supporting information at journals.iucr.org/j

\section{A simple correction for the parallax effect in X-ray pair distribution function measurements}

\author{
Frederick Marlton, ${ }^{\text {a }}$ Oleh Ivashko, ${ }^{\text {b }}$ Martin v. Zimmerman,, Olof Gutowski, \\ Ann-Christin Dippel ${ }^{\mathrm{b}}$ and Mads Ry Vogel Jørgensen ${ }^{\mathrm{a}, \mathrm{c} *}$
}

${ }^{a}$ Center for Materials Crystallography, Department of Chemistry and iNANO, Aarhus University, Langelandsgade, Aarhus, 8000, Denmark, ${ }^{\mathbf{b}}$ Deutsches Elektronen-Synchrotron DESY, Notkestrasse 85, Hamburg, D-22607, Germany, and ${ }^{\mathbf{c}} \mathrm{MAX}$ IV Laboratory, Lund, 22592, Sweden. *Correspondence e-mail: mads@chem.au.dk

Total scattering and pair distribution function (PDF) analysis has created new insights that traditional powder diffraction methods have been unable to achieve in understanding the local structures of materials exhibiting disorder or complex nanostructures. Care must be taken in such analyses as subtle and discrete features in the PDF can easily be artefacts generated in the measurement process, which can result in unphysical models and interpretation. The focus of this study is an artefact called the parallax effect, which can occur in area detectors with thick detection layers during the collection of X-ray PDF data. This effect results in high- $Q$ peak offsets, which subsequently cause an $r$-dependent shift in the PDF peak positions in real space. Such effects should be accounted for if a truly accurate model is to be achieved, and a simple correction that can be conducted via a Rietveld refinement against the reference data is proposed.

\section{Introduction}

Determination of the atomic structure is fundamental in understanding and engineering unique functional materials (Cheetham \& Goodwin, 2014). Structural disorder has become a key aspect in such materials, but understanding such features can be challenging. Total scattering and pair distribution function (PDF) analysis has served as an excellent tool for understanding the local structures of disordered materials, offering new insights that traditional powder diffraction methods have been unable to achieve (Hou et al., 2018; Keen \& Goodwin, 2015; Young \& Goodwin, 2011; Proffen \& Kim, 2009; Petkov, 2008; Billinge, 2008, 2019; Egami, 2007). This field has been rapidly evolving with the developments in computing power, brighter radiation sources and detector efficiencies, allowing more complex experiments and modelling to be achieved (Eremenko et al., 2017; Jiang et al., 2018; Jensen et al., 2012, 2015; Usher et al., 2015; Hu et al., 2013; Roelsgaard et al., 2019; Pramanick et al., 2018).

PDF analysis of atomic structures is typically conducted via small- (Farrow et al., 2007; Coelho et al., 2015) or big-box modelling (Matthew et al., 2007; Gereben et al., 2007; Gereben \& Pusztai, 2012). There have also been developments in complex modelling, which involves analysing different features, such as particle shapes or stacking faults (Juhás et al., 2015; Neder \& Proffen, 2008; Usher et al., 2018). In all modelling methods, the analysis of subtle and discrete features of the PDF is key (Božin et al., 2011; Usher et al., 2016; Thomä et al., 2019). This can include small peaks that give an indication of new bonds or $r$-dependent features that can be 
uncovered by 'box-car' methods (Smith et al., 2008; Proffen \& Kim, 2009; Usher et al., 2016). However, it is important to proceed with caution when analysing such features, as artefacts can easily be generated in the process of obtaining the PDF. There have been various studies that have shown how small discrepancies in the total scattering data can affect the PDF and therefore produce unphysical models and interpretation (Olds et al., 2018; Owen et al., 2017; Toby \& Egami, 1992). The focus of this study is one such artefact that can occur in area detectors with thick detection layers during the collection of X-ray PDF data. In this particular case we apply amorphous silicon flat-panel detectors, but the conclusions are valid for all area detectors with thick detection layers, for example hybrid pixel area detectors.

Despite their shortcomings, flat-panel detectors are today the most commonly used detectors for collecting total scattering data at synchrotron beamlines. This is mainly because of their large area, reasonably fast read-out and relatively low price. The large area allows for rapid collection of high- $Q$ data with good statistics using a single detector position (Chupas et $a l ., 2003)$. It has been noted in previous work that the thickness of the scintillator combined with incomplete X-ray absorption creates artefacts in the diffraction data at high $Q$ (Daniels \& Drakopoulos, 2009). One is called the oblique incidence effect (Zaleski et al., 1998; Skinner et al., 2012; Bendert et al., 2013) and the other the parallax effect (Kawaguchi et al., 2017; Chen et al., 2019; Mendenhall et al., 2015; Pinto et al., 2009). In the former, the effective thickness of the scintillator increases when the photons hit the detector at an oblique angle, leading to an increase in observed

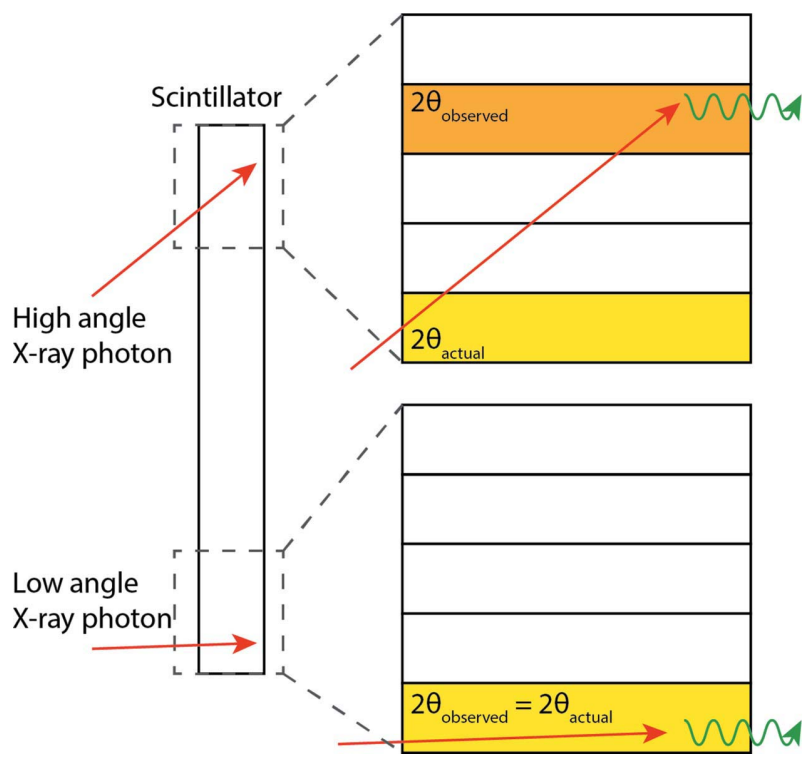

Figure 1

Illustration of the parallax effect in flat-panel detectors for a single X-ray photon. At low scattering angles, the observed $2 \theta$ is the same as the actual $2 \theta$. However, at high angles, the X-ray photon may traverse several pixels, potentially resulting in a difference between the observed and actual $2 \theta$ value. For a large number of scattered photons this will result in a broadening and shift in the centre of mass of the observed peak. intensity. The parallax effect leads to a change in observed peak position. This occurs as the photon is not necessarily detected in the pixel where it hit the front of the detector. At high angles a photon hitting the detector may traverse several pixels before being detected (Daniels \& Drakopoulos, 2009), as illustrated in Fig. 1. Ideally, both of these effects should be corrected directly in the integration software. To our knowledge, only the oblique incidence correction is currently available and well accounted for in commonly used integration software (Filik et al., 2017; Prescher \& Prakapenka, 2015). The parallax effect is in general minor, but it has been suggested that it may be advantageous to correct for it in order to obtain accurate crystalline PDF measurements (Daniels \& Drakopoulos, 2009); however, the effect has to our knowledge never been studied.

In this work we focus on the parallax effect. We show how the resultant high- $Q$ peak offsets affect the corresponding PDF and present a simple solution for correcting them. This has been demonstrated using $\mathrm{Ni}, \mathrm{CeO}_{2}$ and $\mathrm{LaB}_{6}$, which are commonly used standards in this field for data reduction and instrumental parameters. Additionally, they are highly crystalline and scatter well out to high $Q$ values, making them well suited for this study.

\section{Method}

$\mathrm{X}$-ray total scattering was performed at beamline P21.1 at PETRA III, Hamburg, Germany ( $E=103 \mathrm{keV}, \lambda=0.1207 \AA)$. $\mathrm{Ni}$ (Alfa Aesar, 99.9\%, 3-7 $\mu \mathrm{m}$ ), $\mathrm{CeO}_{2}$ (Sigma Aldrich, 99.9\%, $<5 \mu \mathrm{m}$ ) and $\mathrm{LaB}_{6}$ (Sigma Aldrich, 99\%, $10 \mu \mathrm{m}$ ) samples were loaded into $1.02 \mathrm{~mm}$ polyimide capillaries and spun whilst measuring in transmission mode with a 2D PerkinElmer XRD1621 flat-panel detector at a sample-to-detector distance of $351.5 \mathrm{~mm}$. The geometry was calibrated in Dioptas (v 0.4.1) (Prescher \& Prakapenka, 2015) using $\mathrm{CeO}_{2}$, refining the sample-detector distance, beam centre, and pitch and yaw detector angles. The data were subsequently integrated using the refined geometry in Dioptas. The PDFs $G(r)$ were calculated using the PDFgetX3 (Juhás et al., 2013) software from the $x P D F$ suite (Yang et al., 2014) with a $Q_{\max }$ of $24 \AA^{-1}$. Rietveld refinements against the diffraction and PDF data were carried out using the software package TOPAS (Coelho, 2018). Ni and $\mathrm{CeO}_{2}$ were refined with the cubic $F m \overline{3} m$ space group, and $\mathrm{LaB}_{6}$ with the cubic $P m \overline{3} m$ space group.

\section{Results and discussion}

Fig. 2(a) shows the Rietveld refinements on the diffraction data of the three different standard materials of $\mathrm{Ni}, \mathrm{CeO}_{2}$ and $\mathrm{LaB}_{6}$, across a $Q$ range of $1-26 \AA^{-1}$. The inset in the top right of the figure shows the region of the high- $Q$ scattered peaks near $18 \AA^{-1}$. These three data sets were refined simultaneously, applying the same peak shape profiles for all three data sets. Lattice parameters and pseudo-Voight peak profiles were refined.

When observing the fits across the whole range, they appear to be reasonable, with the main differences occurring at the 


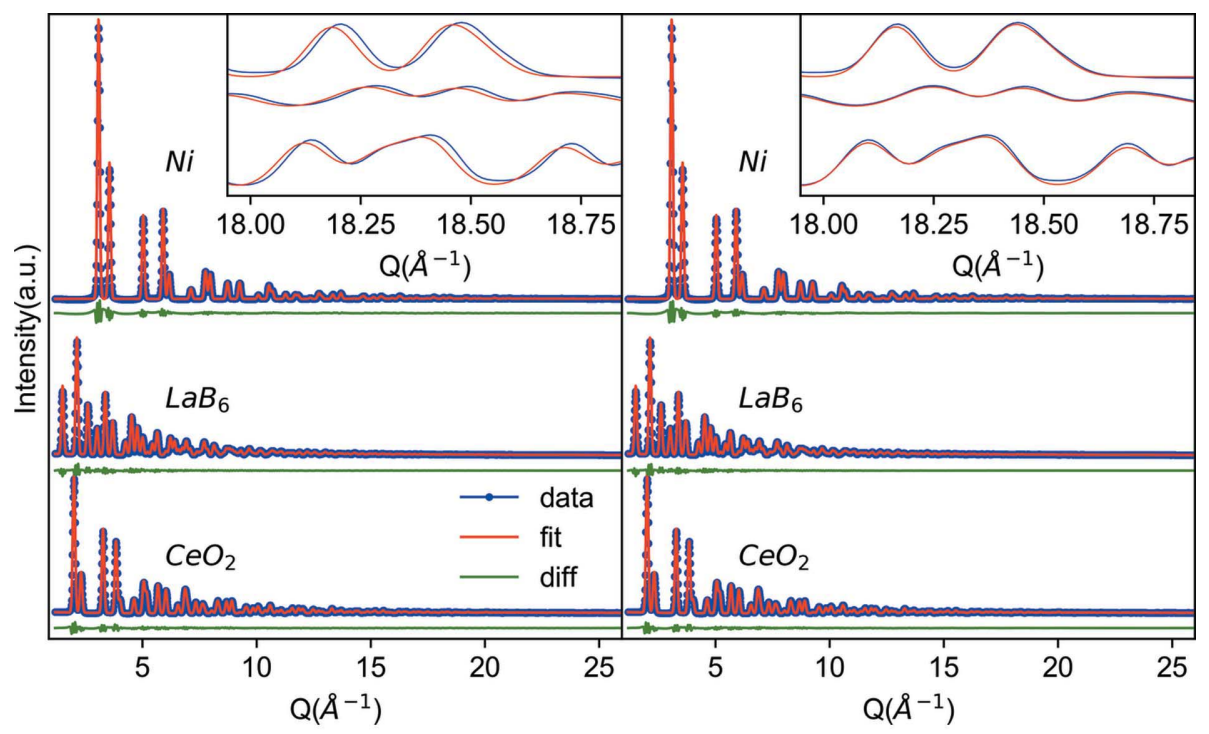

(a)

Figure 2

Rietveld refinements on diffraction data for the $\mathrm{Ni}, \mathrm{CeO}_{2}$ and $\mathrm{LaB}_{6}$ standards. The data are shown in blue, the fit in red and the difference in green. The fits in $(a)$ are with the raw integrated data, whilst in $(b)$ an offset polynomial has been applied. The insets in the top right of $(a)$ and $(b)$ show the region of the high- $Q$ diffraction peaks near $18 \AA^{-1}$.

low- $Q$ high-intensity peaks. Towards high $Q$ values, the difference between the fit and the observed data seems negligible. However, when examining this region in detail it becomes clear that there are some subtle differences between the data and fit [see inset in Fig. 2(a)]. For all three samples, there is a mismatch between the peak positions in the data and fit. The measured peak positions at high $Q$ were all observed at slightly higher $Q$ values than predicted, which is attributed to the parallax effect. This is similar to the observations reported by Daniels \& Drakopoulos (2009), which showed increased shifts in the peak positions for higher $Q$ values. In their study, a correction based on their particular setup was presented. Here, we propose a simple approximation applicable for all detectors perpendicular to the incoming beam.

To correct for these high- $Q$ discrepancies, a quadratic polynomial offset of the form $\Delta 2 \theta=a(2 \theta)^{2}+b(2 \theta)$ was fitted to the data, where $a$ and $b$ are refinable coefficients. These coefficients will vary depending on the beam energy, scintillator material and incident angle. This can easily be implemented in, for example, TOPAS (Coelho, 2018), and an example input script has been included in the supplementary information. This polynomial is a function of $Q$ and hence the offset varies from low $Q$ to high $Q$, which means that the fit can be improved for all $Q$ values. Similar to the peak shapes, the coefficients of this $Q$-dependent offset correction were refined across all three data sets simultaneously. Fig. 2(b) shows the same Rietveld refinement as Fig. 2(a), but this time with the offset polynomial applied.

The high- $Q$ region is shown in the inset of Fig. 2(b), which illustrates that there is a much better agreement between the peak positions of the fit and the measured data after application of the correction. At a $Q$ value of $25 \AA^{-1}$, this corre- sponds to a $Q$ shift of $0.07 \AA^{-1}$, equal to $0.6 \mathrm{~mm}$ ( $\sim 3$ pixels) on the face of the detector. The refined coefficients (supplementary Table S1) of the polynomial are subsequently used to correct the $Q$ values of the measured data.

The goal of collecting high- $Q$ diffraction data is often not to analyse reciprocal-space data but to analyse the calculated PDF. Hence, such small effects can easily go unnoticed and the issue remains as to whether the parallax effect makes any significant difference to the PDF. Fig. 3 shows the PDFs that were calculated for the data with the unmodified $Q$ axis (blue) from Fig. 2(a) and the corrected $Q$ axis (red) and the difference between them (green). In this figure the whole PDF across an $r$ range of $0-45 \AA$ is shown, with insets in the top-left and -right corners showing close-up regions of the low- $r$ and high- $r$ peaks.

Like the diffraction data, the PDFs

in Fig. 3 with and without the correction appear to be very similar when observing the full $r$ range. When observing the low- $r$ peaks near $4 \AA$ in the inset in the top left, the peaks almost completely overlap, with the correction making negligible difference. However, the inset in the top right shows that the peaks at the higher $r$ range near 25-26 $\AA$ have a much larger mismatch in their positions. Observing the peak maxima of $\mathrm{CeO}_{2}$, there is a shift of approximately 0.01 and $0.05 \AA$ at 3.8 and $25 \AA$, respectively.



Figure 3

Comparison between the PDFs calculated without and with the corrected diffraction data across an $r$ range of $0-45 \AA$. The unmodified data are in blue, whilst the corrected data are in red and their difference is in green. Insets in the top-left and -right corners show close-up regions of the low-r and high- $r$ peaks. 
Table 1

$\mathrm{PDF}$ and Bragg refinement parameters for the $\mathrm{Ni}, \mathrm{CeO}_{2}$ and $\mathrm{LaB}_{6}$ samples with and without corrections.

\begin{tabular}{lrllll}
\hline & \multicolumn{2}{l}{ PDF } & & & Bragg \\
\cline { 2 - 3 } \cline { 5 - 6 } Sample & \multicolumn{1}{c}{$R_{\text {wp }}$} & Lattice parameter & & $R_{\text {wp }}$ & Lattice parameter \\
\cline { 6 - 7 } $\mathrm{Ni}$ & 10.048 & $3.52179(7)$ & & 4.269 & $3.52329(12)$ \\
$\mathrm{Ni}-$ corrected & 7.388 & $3.52699(7)$ & & 4.184 & $3.52800(12)$ \\
$\mathrm{LaB}_{6}$ & 16.304 & $4.15300(5)$ & & 3.155 & $4.15532(32)$ \\
$\mathrm{LaB}_{6}-$ corrected & 14.377 & $4.15910(4)$ & & 3.118 & $4.16000(31)$ \\
$\mathrm{CeO}_{2}$ & 12.501 & $5.40675(7)$ & & 3.126 & $5.40777(27)$ \\
$\mathrm{CeO}_{2}-$ corrected & 11.005 & $5.41535(6)$ & & 3.049 & $5.41515(26)$ \\
\hline
\end{tabular}

Such small differences might not appear to be detrimental to the modelling, but previous studies have shown that small artefacts in the data can produce large differences in big-box modelling (Owen et al., 2017).

Rietveld-type refinements against each of the PDFs from 1 to $50 \AA$ were conducted whilst allowing the lattice parameters to refine to further quantify the effect of the correction. The results from this, along with the results from refinements against the diffraction data, are shown in Table 1. It can be seen that the PDF $R_{\mathrm{wp}}$ parameter improves significantly after the inclusion of the parallax correction, indicating that the fit is better in the full $r$ range. In the case of the reciprocal-space refinements, the improvement to $R_{\mathrm{wp}}$ is only minor, as the offset affects the high- $Q$ low-intensity peaks. When comparing

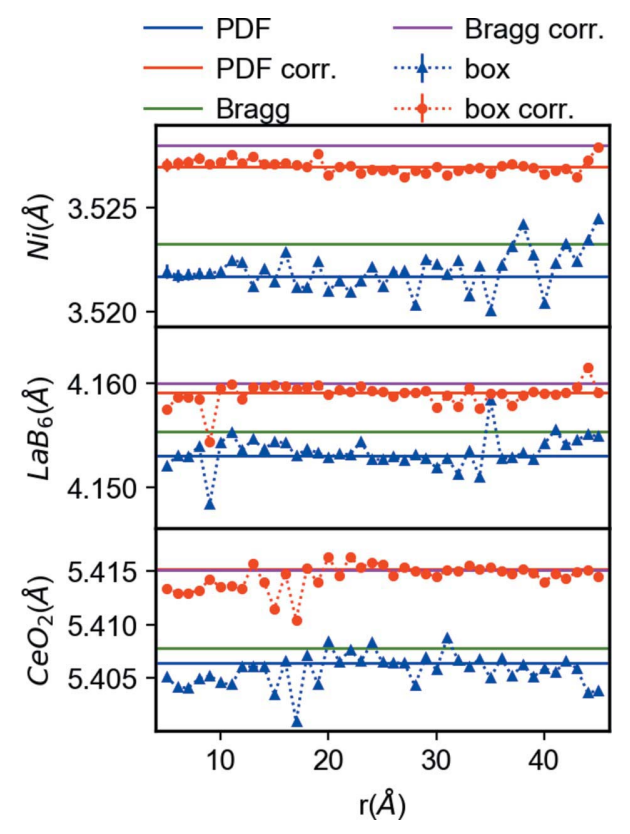

Figure 4

Lattice parameters from refining the Bragg and PDF data. The blue and red solid lines, labelled PDF and PDF corr., correspond to fitting the PDF with an $r$ range of $1-50 \AA$ for the unmodified and corrected data, respectively. The green and magenta lines, labelled Bragg and Bragg corr., correspond to the results from Figs. 2(a) and 2(b), respectively. The red circles and blue triangles, joined by dashed lines and labelled box and box corr., correspond to the 'box-car' results for the unmodified and corrected data, respectively. In the 'box-car' method, a $10 \AA$ window was fitted to the data in $1 \AA$ increments. The $x$ position of the markers corresponds to the centre of the box and the vertical lines correspond to the error bars, which are smaller than the size of the markers. the lattice parameters between the powder X-ray diffraction and PDF refinements across the same data sets (i.e. the same row) in Table 1, it can be seen that there are small differences. These are most noticeable in the $\mathrm{LaB}_{6}$ and Ni samples, which is a known artefact (Jiang et al., 2018; Hong et al., 2016). However, this difference decreases after the correction is applied, which is most notable for $\mathrm{CeO}_{2}$. The difference between the lattice parameters for the fits to the data with and without the correction (i.e. the same column) is much larger, with the corrected data yielding larger unit cells.

The differences between the corrected and uncorrected PDFs increase with $r$, and thus it is expected that a single unit cell cannot fit the data in the full $r$ range. This is tested by conducting a 'box-car' refinement across the PDF fitting range with a $10 \AA$ window and $1 \AA$ increments. The results for all these different fits are summarized in Fig. 4.

Most noticeable in Fig. 4 is the variation in the lattice parameters. For all three samples there is a significantly larger variation across the $r$ range without the parallax correction. When the correction is applied, the lattice parameter is a lot more consistent across the $r$ range. This is further evidence that the lack of the correction results in unphysical $r$-dependent structural variations that can yield incorrect models.

\section{Conclusions}

Total scattering methods are powerful and useful techniques for understanding how subtle structural deviations affect materials properties, but with such minute features being analysed, great care needs to be taken to avoid unphysical artefacts. We have demonstrated how the parallax effect leads to high- $Q$ peak offsets, which subsequently cause an $r$-dependent shift in the PDF peak positions in real space. It is easy to overlook these effects as many PDF analyses focus mainly on the real-space analysis, with small deviations in the high- $Q$ Bragg data easily ignored. Such effects should be accounted for if a truly accurate model is to be achieved. Ideally the raw area-detector data should be corrected for parallax effects. Deconvoluting the effect at this stage would make the correction applicable to experiments where the detector is not normal to the beam. The correction proposed here is empirical and only tested for scenarios where the beam is perpendicular to the detector.

As shown above, erroneous $r$-dependent unit-cell parameters may be observed in 'box-car' refinements if no correction for the parallax effect is applied. These effects are small and may not drastically alter the analysis of amorphous or nanoparticle systems over small $r$ ranges of $0-10 \AA$, where the $r$-dependent peak offset between the two data sets is minimal. However, in the case of crystalline materials this may be an important effect to account for. One example may be ferroelectric relaxors, where many studies have used $r$-dependent refinements, such as the 'box-car' approach, to address deviations between local and average structures (Hou et al., 2018). We propose to correct for this effect by applying a quadratic polynomial offset correction in a Rietveld refinement against the standard reference material (e.g. $\mathrm{CeO}_{2}, \mathrm{LaB}_{6}$ 
etc.) data and subsequently applying this correction to the scattering data of the samples of interest before calculating the PDF.

\section{Acknowledgements}

Affiliation with the Center for Integrated Materials Research (iMAT) at Aarhus University is gratefully acknowledged. We gratefully acknowledge beam time at beamline P21.1 at the PETRA III synchrotron, Deutsches Elektronen-Synchrotron DESY, Hamburg, a member of the Helmholtz Association (HGF), and the great support provided by the beamline team and infrastructure group.

\section{Funding information}

FM and MRVJ are grateful for support by the Danish National Research Foundation (DNRF93), and thank the Danish Agency for Science, Technology and Innovation for funding the instrument center DanScatt.

\section{References}

Bendert, J. C., Mauro, N. A. \& Kelton, K. F. (2013). J. Appl. Cryst. 46, 999-1007.

Billinge, S. J. L. (2008). J. Solid State Chem. 181, 1695-1700.

Billinge, S. J. L. (2019). Philos. Trans. R. Soc. A, 377, 20180413.

Božin, E. S., Masadeh, A. S., Hor, Y. S., Mitchell, J. F. \& Billinge, S. J. L. (2011). Phys. Rev. Lett. 106, 045501.

Cheetham, A. K. \& Goodwin, A. L. (2014). Nat. Mater. 13, 760-762.

Chen, S., Liu, H., Liu, Q., Zheng, Y., Wang, B., Huang, W., Dong, Y., Rong, Y., Jiao, X., Guan, Y., Wang, J., Li, M., Liu, J. \& Zhang, M. (2019). J. Synchrotron Rad. 26, 83-88.

Chupas, P. J., Qiu, X., Hanson, J. C., Lee, P. L., Grey, C. P. \& Billinge, S. J. L. (2003). J. Appl. Cryst. 36, 1342-1347.

Coelho, A. A. (2018). J. Appl. Cryst. 51, 210-218.

Coelho, A. A., Chater, P. A. \& Kern, A. (2015). J. Appl. Cryst. 48, 869-875.

Daniels, J. E. \& Drakopoulos, M. (2009). J. Synchrotron Rad. 16, $463-$ 468.

Egami, T. (2007). Annu. Rev. Mater. Res. 37, 297-315.

Eremenko, M., Krayzman, V., Gagin, A. \& Levin, I. (2017). J. Appl. Cryst. 50, 1561-1570.

Farrow, C. L., Juhas, P., Liu, J. W., Bryndin, D., Božin, E. S., Bloch, J. Th. P., Proffen, T. \& Billinge, S. J. L. (2007). J. Phys. Condens. Matter, 19, 335219.

Filik, J., Ashton, A. W., Chang, P. C. Y., Chater, P. A., Day, S. J., Drakopoulos, M., Gerring, M. W., Hart, M. L., Magdysyuk, O. V., Michalik, S., Smith, A., Tang, C. C., Terrill, N. J., Wharmby, M. T. \& Wilhelm, H. (2017). J. Appl. Cryst. 50, 959-966.

Gereben, O., Jóvári, P., Temleitner, L. \& Pusztai, L. (2007). J. Optoelectron. Adv. Mater. 9, 3021-3027.

Gereben, O. \& Pusztai, L. (2012). J. Comput. Chem. 33, 2285-2291.

Hong, X., Ehm, L., Zhong, Z., Ghose, S., Duffy, T. S. \& Weidner, D. J. (2016). Sci. Rep. 6, 21434.

Hou, D., Zhao, C., Paterson, A. R., Li, S. \& Jones, J. L. (2018). J. Eur. Ceram. Soc. 38, 971-987.

Hu, Y.-Y., Liu, Z., Nam, K.-W., Borkiewicz, O. J., Cheng, J., Hua, X., Dunstan, M. T., Yu, X., Wiaderek, K. M., Du, L.-S., Chapman,
K. W., Chupas, P. J., Yang, X.-Q. \& Grey, C. P. (2013). Nat. Mater. 12, 1130-1136.

Jensen, K. M. Ø., Christensen, M., Juhas, P., Tyrsted, C., Bøjesen, E. D., Lock, N., Billinge, S. J. L. \& Iversen, B. B. (2012). J. Am. Chem. Soc. 134, 6785-6792.

Jensen, K. M. Ø., Yang, X., Laveda, J. V., Zeier, W. G., See, K. A., Michiel, M. D., Melot, B. C., Corr, S. A. \& Billinge, S. J. L. (2015). J. Electrochem. Soc. 162, A1310-A1314.

Jiang, B., Raeder, T. M., Lin, D.-Y., Grande, T. \& Selbach, S. M. (2018). Chem. Mater. 30, 2631-2640.

Juhás, P., Davis, T., Farrow, C. L. \& Billinge, S. J. L. (2013). J. Appl. Cryst. 46, 560-566.

Juhás, P., Farrow, C., Yang, X., Knox, K. \& Billinge, S. (2015). Acta Cryst. A71, 562-568.

Kawaguchi, S., Takemoto, M., Osaka, K., Nishibori, E., Moriyoshi, C., Kubota, Y., Kuroiwa, Y. \& Sugimoto, K. (2017). Rev. Sci. Instrum. 88, 085111.

Keen, D. A. \& Goodwin, A. L. (2015). Nature, 521, 303-309.

Matthew, G. T., David, A. K., Martin, T. D., Andrew, L. G. \& Qun, H. (2007). J. Phys. Condens. Matter, 19, 335218.

Mendenhall, M. H., Mullen, K. \& Cline, J. P. (2015). J. Res. Natl Inst. Stand. Technol. 120, 223-251.

Neder, R. B. \& Proffen, T. (2008). Diffuse Scattering and Defect Structure Simulations: A Cook Book Using the Program DISCUS. Oxford University Press.

Olds, D., Saunders, C. N., Peters, M., Proffen, T., Neuefeind, J. \& Page, K. (2018). Acta Cryst. A74, 293-307.

Owen, L. R., Playford, H. Y., Stone, H. J. \& Tucker, M. G. (2017). Acta Mater. 125, 15-26.

Petkov, V. (2008). Mater. Today, 11, 28-38.

Pinto, S. D., Villa, M., Alfonsi, M., Brock, I., Croci, G., David, E., Oliveira, R., Ropelewski, L., Stenis, M. \& Taureg, H. (2009). J. Instrum. 4, P12006-P12006.

Pramanick, A., Dmowski, W., Egami, T., Budisuharto, A. S., Weyland, F., Novak, N., Christianson, A. D., Borreguero, J. M., Abernathy, D. L. \& Jørgensen, M. R. V. (2018). Phys. Rev. Lett. 120, 207603.

Prescher, C. \& Prakapenka, V. B. (2015). High. Pressure Res. 35, 223 230.

Proffen, T. \& Kim, H. (2009). J. Mater. Chem. 19, 5078-5088.

Roelsgaard, M., Dippel, A.-C., Borup, K. A., Nielsen, I. G., Broge, N. L. N., Röh, J. T., Gutowski, O. \& Iversen, B. B. (2019). IUCrJ, 6, 299-304.

Skinner, L. B., Benmore, C. J. \& Parise, J. B. (2012). Nucl. Instrum. Methods Phys. Res. A, 662, 61-70.

Smith, M. B., Page, K., Siegrist, T., Redmond, P. L., Walter, E. C., Seshadri, R., Brus, L. E. \& Steigerwald, M. L. (2008). J. Am. Chem. Soc. 130, 6955-6963.

Thomä, S. L. J., Krauss, S. W., Eckardt, M., Chater, P. \& Zobel, M. (2019). Nat. Commun. 10, 995.

Toby, B. H. \& Egami, T. (1992). Acta Cryst. A48, 336-346.

Usher, T.-M., Iamsasri, T., Forrester, J. S., Raengthon, N., Triamnak, N., Cann, D. P. \& Jones, J. L. (2016). J. Appl. Phys. 120, 184102.

Usher, T.-M., Levin, I., Daniels, J. E. \& Jones, J. L. (2015). Sci. Rep. 5, 14678.

Usher, T.-M., Olds, D., Liu, J. \& Page, K. (2018). Acta Cryst. A74, 322 331.

Yang, X., Juhas, P., Farrow, C. L. \& Billinge, S. J. (2014). arXiv: 1402.3163

Young, C. A. \& Goodwin, A. L. (2011). J. Mater. Chem. 21, 64646476.

Zaleski, J., Wu, G. \& Coppens, P. (1998). J. Appl. Cryst. 31, 302-304. 